\title{
Robust adaptive beamforming method for active sonar in single snapshot
}

\author{
Xu Sun ${ }^{*}$, Ranwei Li \\ Science and Technology on Sonar Laboratory, Hangzhou Applied Acoustics Research Institute, Hangzhou, China
}

\begin{abstract}
Forming narrow beams is a useful way for active sonar to anti-reverberation when it works in the shallow water. High-resolution adaptive beamforming with the performance of narrow beamwidths and low sidelobe levels is a better and more efficient method, particularly in the scenario where the installation space for sonar array is limited, such as hull-mounted sonar. Due to the short duration of target echo signal in the complex and varying acoustic channel, conventional adaptive beamforming methods are invalid. Therefore, this paper proposes a robust adaptive beamforming method for active sonar in single snapshot, also called the steered dominant mode rejection (STDMR). Firstly, STDMR steered the sample covariance matrix (STCM) based on wide-band focusing, which the needed number of snapshots is greatly reduced. Secondly, by partial eigendecomposition, the large eigenvalues of the STCM which are greater than the noise energy and their eigenvectors are used for dominant mode rejection (DMR). DMR is a typical eigenspace-based algorithm which has small computational load and fast convergence speed. Finally, modified with the methods of diagonal loading of $3-5 \mathrm{~dB}$ over the noise energy and signal mismatch protection, improved the robustness of this method. Simulation and experimental data analysis shows that the STDMR method achieves narrow beams and low-level sidelobes in single snapshot. Hence, the STDMR beamformer is an appropriate implementation to use for active sonar detection systems.
\end{abstract}

\section{Introduction}

Conventional beamForming ( $\mathrm{CBF})$ has been widely used in sonar due to its robustness and less computational complexity. However, its low spatial resolution and high sidelobe level are often cause weak target signal to be submerged in energy leakage of strong interferences, and its poor ability for reverberation suppression. Then, adaptive beamforming (ABF) methods were intorduced to overcome those deficiencies of $\mathrm{CBF}$, which could form notches at directions of interferences in the beampattern of specified direction.

In 1969, the minimum variance distortionless response (MVDR) adaptive beamforming method was first proposed by Capon, which is applied to seismic signal processing[1]. The notch performance of MVDR is based on the accurate estimation of cross-spectral density matrix (CSDM) of the actual underwater acoustic field, which requires the acoustic field to be stable over a long time. Thus, MVDR cannot be applied in pulse signal detection in complex and variable oceans for active sonar. Further, Swingler proposed the Steered minimum variance (STMV) distortionless response adaptive beamforming algorithm[2], which extends the narrowband MVDR into the wideband signal and uses the wideband coherent processing to reduce the needed number of snapshots. However, due to the signal mismatch and beampattern distortion in actual data,
STMV is difficult to achieve ideal spatial resolution and sidelobe level, and its performance will even be inferior to $\mathrm{CBF}[3]$. A mismatch protection methods in adaptive beamforming was proposed by Dr. Henry Cox[8], which is suitable for high signal-to-noise ratio occasions. $\mathrm{Li}$ Jian proposed an optimal estimation for diagonal loading value[4] to overcome the influence of small eigenvalues in CSDM, but it is difficult to determine the error ellipse of the steering vector.

In the complex and variable ocean channel, robust adaptive beamforming for active sonar is difficult to achieve; for instance, the poor phase consistency of transducer (resonant emission) in the hull-mounted sonar, or the position error of hydrophone array in the towed sonar. To solve the above problem, this paper proposes a robust adaptive beamforming method for active sonar in single snapshot, also called the steered dominant mode rejection (STDMR), which based on the dominant mode rejection (DMR) algorithm[5-7]. STDMR can effectively adjust beampattern according to the actual acoustic field in real time, notches are aligned in the interferences direction, and the weak signals are protected from the energy leakage of strong interferences.

\section{Conventional adaptive beamforming}

* Corresponding author: $1513638732 @$ qq.com 
As one of the basic conventional adaptive beamforming, the MVDR represents that the total output power of the array is minimized with the constraint of signal in specified beam direction passed through without distortion, expressed as

$$
\begin{gathered}
\min _{\mathbf{w}(\theta, f)} \mathbf{w}^{\mathrm{H}}(\theta, f) \mathbf{R}(f) \mathbf{w}(\theta, f) \\
\text { s.t. } \quad \mathbf{w}^{\mathrm{H}}(\theta, f) \mathbf{a}(\theta, f)=1
\end{gathered}
$$

where $\mathbf{w}(\theta, f)$ is the weight vector of beamforming at direction $\theta$ in frequency unit $f ; \mathrm{H}$ is the conjudate transposed matrix; $\mathbf{R}(f)$ is the cross-spectral density matrix of frequency unit $f ; \mathbf{X}(f)$ is the array snapshot data corresponding with the frequency unit $f ; \mathbf{a}(\theta, f)$ is the CBF weight vector, which is given by

$$
a_{n}(\theta, f)=\exp \left[-j 2 \pi f \tau_{n}(\theta)\right], n=1,2, \cdots, N
$$

where $\tau_{n}(\theta)$ is the arrival time delay of the $\theta$ beam direction at the nth number element; $N$ is the number of sonar arrays.

In mathematical, there is a constrained minimization problem solves the weight vector of MVDR. Lagrange method can be used to construct the cost function as follows

$$
\begin{gathered}
L[\mathbf{w}(\theta, f)]=\mathbf{w}^{\mathrm{H}}(\theta, f) \mathbf{R}(f) \mathbf{w}(\theta, f) \\
+\lambda\left[1-\mathbf{w}^{\mathrm{H}}(\theta, f) \mathbf{a}(\theta, f)\right]
\end{gathered}
$$

Derivate the cost function $L[\mathbf{w}(\theta, f)]$ on $\mathbf{w}$ and make it equal to zero. Then, the best weight vector is obtained by

$$
\mathbf{w}(\theta, f)=\frac{\lambda}{2} \mathbf{R}^{-1}(f) \mathbf{a}(\theta, f)
$$

Constant $\lambda$ can be eliminated by (1) and (4), then

$$
\mathbf{w}(\theta, f)=\frac{\mathbf{R}^{-1}(f) \mathbf{a}(\theta, f)}{\mathbf{a}^{\mathrm{H}}(\theta, f) \mathbf{R}^{-1}(f) \mathbf{a}(\theta, f)}
$$

Generally, MVDR requires the underwater acoustic field to be stable over a long time. However, the condition is difficult to satisfied in the complex and dynamic ocean environment for echo signal detection. STMV was first proposed by Swingler, which utilizes the space-time characteristics of the underwater acoustic field for wide-band coherent processing. The space-time statistical covariance matrix (STCM) is written as

$$
\begin{aligned}
\mathbf{R}_{S}(\theta)= & \sum_{k=1}^{K} \mathbf{T}\left(f_{k}, \theta\right) \mathbf{R}\left(f_{k}\right) \mathbf{T}^{\mathrm{H}}\left(f_{k}, \theta\right) \\
& =\sum_{k=1}^{K} \mathbf{Y}\left(f_{k}\right) \mathbf{Y}^{\mathrm{H}}\left(f_{k}\right)
\end{aligned}
$$

where $\mathbf{T}\left(f_{k}, \theta\right)$ is a diagonal matrix as follows

$$
\begin{gathered}
\mathbf{T}\left(f_{k}, \theta\right)=\operatorname{diag}\left\{\exp \left[j 2 \pi f_{k} \tau_{1}(\theta)\right],\right. \\
\exp \left[j 2 \pi f_{k} \tau_{2}(\theta)\right] \cdots, \exp \left[j 2 \pi f_{k} \tau_{N}(\theta)\right]
\end{gathered}
$$

where $f_{k}$ denotes the frequency bin, $k=1,2, \cdots, K$ is the subscript mark the corresponding frequency; $\mathbf{Y}\left(f_{k}\right)$ is the snapshot data.

The weight vector of STMV is derived as

$$
\mathbf{w}_{S}(\theta)=\frac{\mathbf{R}_{S}^{-1}(\theta) \mathbf{e}}{\mathbf{e}^{\mathrm{H}} \mathbf{R}_{S}^{-1}(\theta) \mathbf{e}}
$$

where $\mathbf{e}$ denotes the unit vector.

After steered processing, no matter what the time frequency $\left(f_{k}\right)$ of a signal, the final spatial frequency $\left(v_{k}=2 \pi f_{k} d \cos (\theta) / c\right)$ is equal to zero for the signal in the direction of the preformed beam, that the broadband coherent processing is realized. And makes the needed number of snapshots for STMV are greatly reduced.

\section{STDMR}

Based on the data analysis of active sonar in the complex and dynamic marine environment shows that, both MVDR and STMV adaptive beamformer are difficult to achieve ideal performance, even less then CBF. The reasons for this phenomenon are lies in the following two points.

Firstly, signal mismatch. MVDR and STMV beamformer are sensitive to signal mismatch. The inconsistency between the beam direction and the echo direction, array perturbation distortion, near-field propagation, non-stationary noise or the limited number of snapshots are leads to the signal mismatch, that all makes the signal beams are lowered in power response, and result in loss of detection performance.

Secondly, small eigenvalue perturbation. In the high SNR or small number of snapshots situations, CSDM and STCM are often ill-conditioned. The large eigenvalues (signal) are much larger than the small eigenvalues (noise), which makes the beam power of MVDR and STMV affected by small eigenvlaues, and result in beam pattern distortion and poor notches performance.

In this paper, STDMR method was proposed, that could effectively solve the above problems to achieve robust high resolution and low sidelobe performance by the convergence rate and robustness improved. Therefore, it could improve the detection ability for echo signal in the shallow reverberation or strong interferences.

\subsection{Convergence acceleration}

In the sampling covariance matrix of acoustics field, the beam power is greatly influenced by the components of large signals or strong interferences. However, the small components have less influence, but their estimation costs are high. Dominant mode rejection was introduced to further improve the convergence speed of the adaptive beamformer.

Eigen-decomposition of $\mathbf{R}_{S}$

$$
\mathbf{R}_{S}=\sum_{i=1}^{D} \lambda_{i} \mathbf{v}_{i} \mathbf{v}_{i}^{\mathrm{H}}+\sum_{i=D+1}^{N} \lambda_{i} \mathbf{v}_{i} \mathbf{v}_{i}^{\mathrm{H}}
$$

where $\mathbf{v}_{i}$ is an eigenvector corresponding to the eigenvalue of $\lambda_{i}, \lambda_{1} \geq \cdots \geq \lambda_{D} \geq \lambda_{D+1} \geq \cdots \geq \lambda_{N} ; D$ is the dimension of dominant mode subspace; $\mathbf{U}_{s}=\left[\mathbf{v}_{1}, \mathbf{v}_{2}, \cdots \mathbf{v}_{D}\right]$ forms the subspace of dominant mode and $\mathbf{U}_{n}=\left[\mathbf{v}_{D+1}, \mathbf{v}_{D+2}, \cdots \mathbf{v}_{N}\right]$ forms the subspace of minor mode.

By only reject the components of dominant mode in STCM, the needed number of snapshots for adaptive beamformer was reduced to $D$ of $N$ times. And the convergence rate had been increased $K$ times (where $K$ is the number of frequency bins) after the pre-steered processing. Then, the number of snapshots required by 
STDMR is $2 N \cdot \frac{1}{K} \cdot \frac{D}{N}$. If $D<\frac{K}{2}$, active adaptive beamforming can be realized in single snapshot.

In order to estimate the dimension of the dominant mode subspace, this paper proposed a criterion based on the noise power of the specified beams, that is

If $\lambda_{i}>\eta_{1} \sigma_{n}^{2}, \lambda_{i}, \mathbf{v}_{i}$ are components of dominant mode;

If $\lambda_{i}<\eta_{1} \sigma_{n}^{2}, \lambda_{i}, \mathbf{v}_{i}$ are components of minor mode. where $\sigma_{n}^{2}$ is the noise power of preformed beam, $\sigma_{n}^{2}$ can be estimate by TPM methods; $\eta_{1}$ is a constant.

\subsection{Robust improvement}

The convergence speed of the algorithm was improved effectively by means of dominant mode rejection, and the influence of small eigenvalue disturbance was partly reduced. In order to further enhance the robustness of adaptive processing, a mismatch protection methods was designed based on the intrinsic relationship between eigenvectors and beam steering vectors; And a simple diagonal loading methods was proposed from the relationship between the optimal diagonal loading and the noise power on the specified beam.

(1) Mismatch protection

Regarding the observation beam of direction $\theta$, the test criterion to choice whether $\mathbf{v}_{i}$ is a signal (on the observation beam) or an interference (from other beams) are as follows

If $\left|\mathbf{v}_{i}^{\mathrm{H}} \mathbf{e}\right|^{2} \leq \gamma, \mathbf{v}_{i}$ is an interference from other beams, STDMR forms a notch for this interference to rejects its energy leakage;

If $\left|\mathbf{v}_{i}^{\mathrm{H}} \mathbf{e}\right|^{2}>\gamma, \mathbf{v}_{i}$ is a signal on the observation beam, there is no need to rejects this component. where $\gamma$ is a constant.
(2) Diagonal loading

Li Jian proposed an optimal adaptive beamforming method based on the steering vector errors satisfies an uncertainty constraint, and it's proved that this method is equivalent to the optimal diagonal loading. The optimization expression is

$$
\left\{\begin{array}{c}
\min _{\mathbf{a}} \mathbf{b}^{\mathrm{H}}(\theta) \mathbf{R}_{s}^{-1}(\theta) \mathbf{b}(\theta) \\
\text { s.t. }\|\mathbf{b}(\theta)-\overline{\mathbf{b}}(\theta)\|^{2} \leq \varepsilon
\end{array}\right.
$$

where $\mathbf{b}(\theta)$ is the steering vector for real signal after pre-steer processing; $\overline{\mathbf{b}}(\theta)$ is the steering vector for the observation beam, $\overline{\mathbf{b}}(\theta)=\boldsymbol{e}$.

In practical processing, the calculation of the optimal diagonal loading was very time-consuming, and the algorithm performance was affected by the selection of constrained parameters. Yet, diagonal loading is order to suppressing the small eigenvalue perturbations, which are often associated with noise. The optimal diagonal load to noise energy ratio (LNR) under different constraint parameters was established in this paper through simulation (16-element half-wavelength-spaced circular array, the pulse width of the active signal is $0.5 \mathrm{~s}$ and the bandwidth of the active signal is $300 \mathrm{~Hz}$ ), as shown in Fig.1. It can be seen from the Fig. 1 that under the background of white noise, the optimal diagonal load is $0-5 \mathrm{~dB}$ higher than the noise power in reasonable range of constraint parameter $(\varepsilon \in[0.3,0.5])$. The LNR increased when signals or interferences influenced. Therefore, the value of a certain times of noise power for diagonal loading was used in this paper, that is

$$
\sigma_{L}^{2}=\eta_{2} \cdot \sigma_{n}^{2}
$$

where $\sigma_{L}^{2}$ is diagonal loading value; $\eta_{2}$ is a constant value.

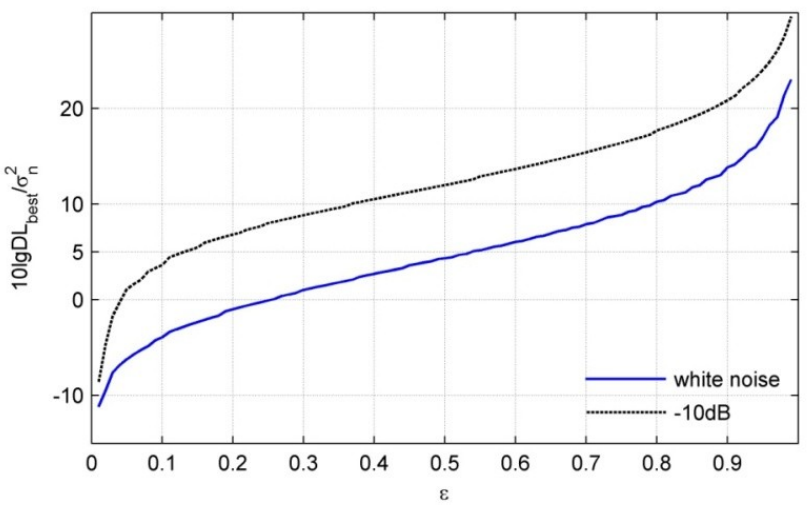

Fig. 1. The optimal diagonal loading to noise energy ratio $\left(10 \lg \left(D L_{b e s t} / \sigma_{n}^{2}\right)\right)$ for different constrained parameters $(\varepsilon)$ on the observed beam

\subsection{Algorithm recursion equations}

Eigen-decomposition of $\mathbf{R}_{S}$, the average eigen-values of minor mode subspace is

$$
\alpha=\frac{1}{N-D} \sum_{i=D+1}^{N} \lambda_{i}=\frac{1}{N-D}\left[\operatorname{tr}\left(\mathbf{R}_{S}\right)-\sum_{i=1}^{D} \lambda_{i}\right]
$$

In an environment of $D$ signals (interferences), $\alpha$ represents the noise power. Then, the dominant mode subspace obtained by eigen-decomposition can be used to instead of $\mathbf{R}_{S}$, that is, $\widehat{\mathbf{R}}_{S}$

$$
\widehat{\mathbf{R}}_{S}=\alpha\left[\mathbf{I}+\sum_{i=1}^{D}\left(\frac{\lambda_{i}-\alpha}{\lambda_{i}}\right) \mathbf{v}_{i} \mathbf{v}_{i}^{\mathrm{H}}\right]
$$

The inverse of $\widehat{\mathbf{R}}_{S}$ can be determined as follows

$$
\widehat{\mathbf{R}}_{S}^{-1}=\alpha^{-1}\left[\mathbf{I}-\sum_{i=1}^{D}\left(\frac{\lambda_{i}-\alpha}{\lambda_{i}}\right) \mathbf{v}_{i} \mathbf{v}_{i}^{\mathrm{H}}\right]
$$

Consider the diagonal loading, that is, 


$$
\left[\widehat{\mathbf{R}}_{S}+\sigma_{L}^{2} \mathbf{I}\right]^{-1}=\left(\alpha+\sigma_{L}^{2}\right)^{-1}\left[\mathbf{I}-\sum_{i=1}^{D}\left(\frac{\lambda_{i}-\alpha}{\lambda_{i}+\sigma_{L}^{2}}\right) \mathbf{v}_{i} \mathbf{v}_{i}^{\mathrm{H}}\right](15)
$$

The weight vector of adaptive beamforming is as follows

$$
\mathbf{w}_{d s}(\theta)=\left(\mathbf{e}-\sum_{i=1}^{D} \beta_{i} \mathbf{v}_{i}^{\mathrm{H}} \mathbf{e} \mathbf{v}_{i}\right) /\left(|\mathbf{e}|^{2}-\sum_{i=1}^{D} \beta_{i}\left|\mathbf{v}_{i}^{\mathrm{H}} \mathbf{e}\right|^{2}\right)
$$

where $\quad \beta_{i} \quad$ are suppressing coefficient, $\beta_{i}=\left(\lambda_{i}-\alpha\right) /\left(\lambda_{i}+\sigma_{L}^{2}\right) ; \mathbf{w}_{d s}(\theta)$ is independent of frequency and can be applied to all frequency units.

A mismatch protection factor $\left(\delta_{i}\right)$ are introduced for mismatch protection

$$
\delta_{i}= \begin{cases}1 & \left|\mathbf{v}_{i}^{\mathrm{H}} \mathbf{e}\right|^{2} \leq \gamma \\ 0 & \left|\mathbf{v}_{i}^{\mathrm{H}} \mathbf{e}\right|^{2}>\gamma\end{cases}
$$

Then, the weight vector can be changed to $\mathbf{w}_{d s}(\theta)=$

$\left(\mathbf{e}-\sum_{i=1}^{D} \delta_{i} \beta_{i} \mathbf{v}_{i}^{\mathrm{H}} \mathbf{e v}\right) /\left(|\mathbf{e}|^{2}-\sum_{i=1}^{D} \delta_{i} \beta_{i}\left|\mathbf{v}_{i}^{\mathrm{H}} \mathbf{e}\right|^{2}\right)$

The output of adaptive beamforming is

$$
B(\theta, f)=\mathrm{w}_{d s}^{\mathrm{H}}(\theta) \mathbf{Y}(\mathrm{f})
$$

\section{Data verification}

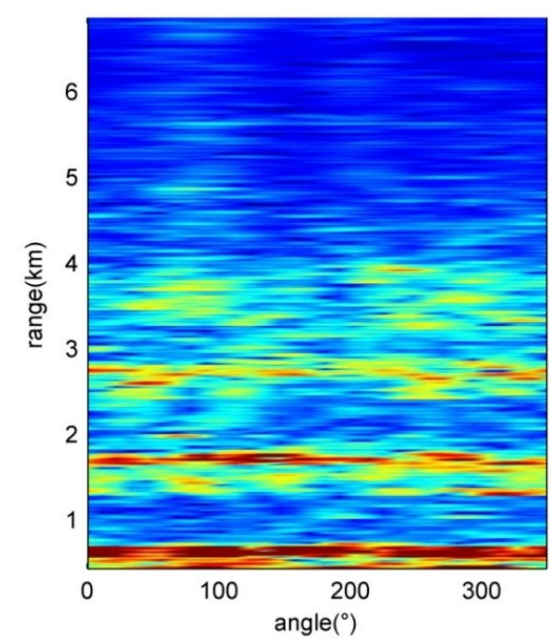

To verify the validity of STDMR algorithm, CBF and STDMR are performed on five consecutive active data of a small sonar array, which collected in the South China Sea.

Fig.2 shows the detection result of the first data. The angle-range display image processed by $\mathrm{CBF}$ is on the left side and the angle-range display image processed by STDMR is on the right side. The bright spot at $2 \mathrm{~km}$ and 68 degrees is the test target. It can be seen that the resolution of STDMR is higher than that of CBF, that is, narrow beam and high resolution processing are realized.

Fig. 3 shows a comparison of the target beam output of the five consecutive detection data in CBF and STDMR. The blue on the left is CBF result and the red on the right is STDMR result. From the figure, we can see that CBF method can detects the echo signal of the first, second and third data, but it can not detects the echo signal in the fourth and fifth data; STDMR method can detects the echo signal well in the first, second, third and fifth data, and has weak detection ability for the fourth data. The results show that STDMR method has better signal detection ability than CBF method under reverberation interference, and the signal beam has no distortion, indicating that STDMR method has the robustness of CBF method.

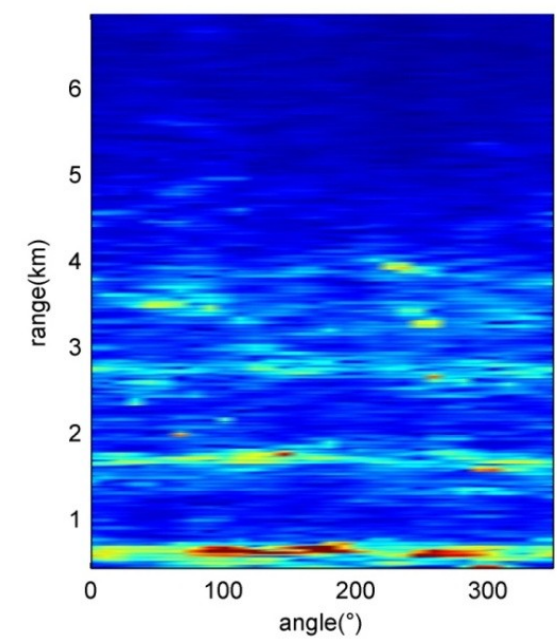

Fig. 2. Comparison of CBF (left) and STDMR (right) processing in the first data.
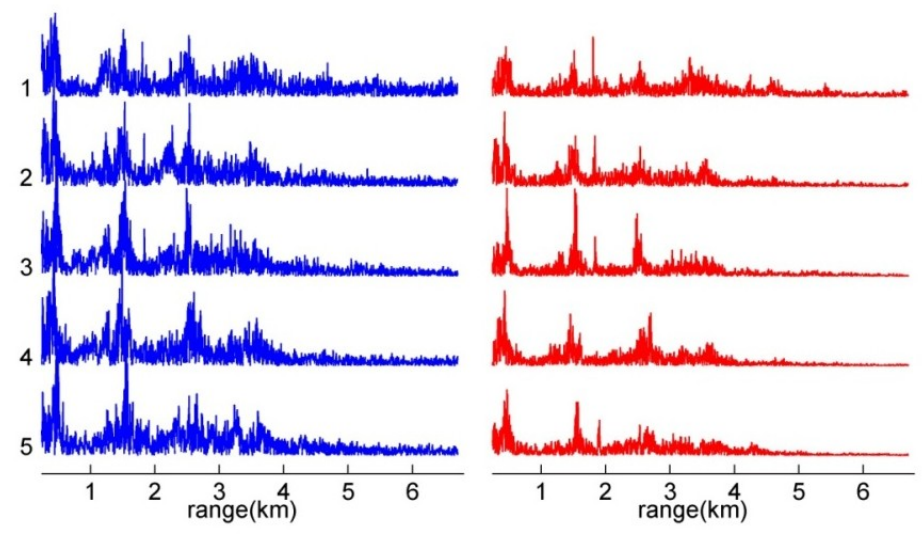

Fig. 3. Continuous five data, CBF (left) and STDMR (right) target beam output comparison

\section{Conclusions}

Through the theoretical analysis of adaptive beamforming technology for active sonar, the 
fundamental reasons (signal mismatch and small eigenvalue perturbation) for the poor performance of MVDR and STMV methods are obtained. Then, it guides the design of STDMR algorithm, and ensures each parameter values are reasonable. Simulation and experimental data processing shows the STDMR has the following advantages,

(1) Excellent snapshot performance. STDMR algorithm achieves high-resolution and low sidelobe levels for active sonar in single snapshot mainly by dominant mode rejection and wide-band focusing.

(2) High robustness, even comparable to CBF. STDMR algorithm was processed robustness through mismatch protection and diagonal loading based on LNR.

(3) Less computational burden. For only eigendecomposition dominant mode of STCM, the computational burden of STDMR is smaller than that of STMV or MVDR methods.

\section{References}

1. Capon J., Proceeding of the IEEE, 57, 8(1969)

2. Swingler N D., IEEE Transactions on signal processing, 47, 4(1999)

3. Li Jian, Stoica P, Wng zhisong, IEEE Transactions on signal processing, 51(2003)

4. Zhou shenzeng, Du xuanmin, Acta Acustica, 34, 6(2009)

5. N L Owsley, Underwater acoustic data processing, 1989

6. Kathleen E. Wage, John R. Buck, IEEE J. Ocean. Eng., 39, 2(2014)

7. John R. Buck, Kathleen E. Wage, IEEE SSP, (2012)

8. Ning Li, Jun Tang, Yingning Peng, Xiutan Wang, IEEE, (2006)

9. H. Cox, R. Pitre, IEEE, 1998

10. Beer S P, van IJsselmuide S P., UDT Europe 2001.

11. I.P. Kirsteins and D.W. Tufts, Proc. ICASSP-85, 1985

12. Stergious Stergiopoulos, Proceedings of the IEEE, 86, 2(1998)

13. C. D. Richmond, IEEE, (2000)

14. Xu, Xiaonan; Ma, Qiming; Du, Shuanping, ACTA ACUSTICA, (2013) 